\title{
Synthesis of Ketenimines Derivatives from Reaction between Alkyl Isocyanides and Dialkyl Acetylenedicarboxylates in the Presence of Carboxylic Acid Arylidene-Hydrazides
}

\author{
ALIREZA HASSANABADI*1 ${ }^{*}$, MOHAMMAD H. MOSSLEMIN ${ }^{2}$ and \\ MOHAMMAD REZA SALARI ${ }^{2}$ \\ ${ }^{1}$ Department of Chemistry, Islamic Azad University, Zahedan Branch, P.O. Box 98135-978, \\ Zahedan, Iran \\ ${ }^{2}$ Department of Chemistry, Islamic Azad University, Yazd Branch, P.O. Box 89195-155, Yazd, Iran \\ ar_hasanabadi@yahoo.com
}

Received 5 May 2012 / Accepted 17 May 2012

\begin{abstract}
An improved three-component reaction of cyclohexyl isocyanide is described. The reaction between alkyl isocyanides and dialkyl acetylenedicarboxylates in the presence of carboxylic acid arylidene-hydrazides to afford highly functionalized ketenimines in good yields. The reaction was characterized by mild conditions, high selectivity and tolerance to various functional groups.
\end{abstract}

Keywords: Ketenimines, Dialkyl acetylenedicarboxylates, Alkyl isocyanides, NH-acids, Threecomponent reaction

\section{Introduction}

Ketenimines have been extensively used in organic synthesis as versatile building blocks for the preparation of a large variety of cyclic compounds via inter- or intramolecular cycloaddition reactions ${ }^{1,2}$. There has been intense interest in their addition reactions, such as cycloaddition $^{3-5}$ Multicomponent reactions (MCRs), by virtue of their convergence, productivity, facile execution and generally high yields of products, have attracted much attention in the area of combinatorial chemistry ${ }^{6-8}$, of pivotal importance are the isocyanidebased MCRs $^{9}$. The trapping of the 1:1 intermediate formed between dialkyl acetylenedicarboxylates and isocyanides with $\mathrm{OH}, \mathrm{NH}$ and $\mathrm{CH}$ acids has been widely studied $^{10-16}$. In continuation of our works on the reaction between isocyanides and dialkyl acetylenedicarboxylates in the presence of organic acids ${ }^{17-19}$, here we wish to report the results of our studies on the reaction between alkyl isocyanides and dialkyl acetylenedicarboxylates, in the presence of carboxylic acid arylidene-hydrazides.

\section{Experimental}

Elemental analyses were performed using a Costech ECS 4010 CHNS-O analyzer at analytical laboratory of Islamic Azad University Yazd Branch ${ }^{1} \mathrm{H}$ and ${ }^{13} \mathrm{CNMR}$ spectra were recorded on BRUKER DRX-500 AVANCE spectrometer in CDCl3 using TMS as internal standard. The chemicals used in this work were purchased from Fluka (Buchs, Switzerland) and were used without further purification. 


\section{Experimental}

To a magnetically stirred solution of dialkyl acetylenedicarboxylate $(2 \mathrm{mmol})$ and carboxylic acid arylidene-hydrazide $(2 \mathrm{mmol})$ in acetone $(10 \mathrm{~mL})$ was added a solution of alkyl isocyanide $(2 \mathrm{mmol})$ in acetone $(5 \mathrm{~mL})$ dropwise at room temperature over 10 minute. The mixture was then allowed to stir for $24 \mathrm{~h}$. The solvent was removed under reduced pressure, and the residue was separated by column chromatography (silica gel, hexane-ethyl acetate, 3:1) to afford the pure titled compounds.

Dimethyl 2-[N'-benzylidene- $N$-(3-methyl)hydrazino]-3- (cyclohexyl iminomethylene) succinate (4a)

Yield: 89\%; Yellow oil; IR (KBr) $\left(v_{\max }, \mathrm{cm}^{-1}\right)$ : $2078(\mathrm{~N}=\mathrm{C}=\mathrm{C}), 1741,1666(\mathrm{C}=\mathrm{O})$. Analyses: Calcd. for $\mathrm{C}_{22} \mathrm{H}_{27} \mathrm{~N}_{3} \mathrm{O}_{5}$ : C, 63.91; H, 6.58; N, 10.16\%. Found: 63.82; H, 5.71; $\mathrm{N}, 10.23 \%$. MS (m/z, \%): $413(\mathrm{M}+, 5) .{ }^{1} \mathrm{H}$ NMR $\left(500.1 \mathrm{MHz}, \mathrm{CDCl}_{3}\right): \delta=1.20-2.01(10$ $\mathrm{H}, \mathrm{m}, 5 \mathrm{CH}_{2}$ of cyclohexyl), $2.33\left(3 \mathrm{H}, \mathrm{s}, \mathrm{CH}_{3}\right), 3.71\left(3 \mathrm{H}, \mathrm{s}, \mathrm{OCH}_{3}\right), 3.79(3 \mathrm{H}, \mathrm{s}$, $\left.\mathrm{OCH}_{3}\right), 3.89(1 \mathrm{H}, \mathrm{m}, \mathrm{CH}$ of cyclohexyl), $5.87(1 \mathrm{H}, \mathrm{s}, \mathrm{CH}), 7.40-7.83(5 \mathrm{H}, \mathrm{m}$, aromatic), $8.32(1 \mathrm{H}, \mathrm{s}, \mathrm{N}=\mathrm{CH}) \mathrm{ppm} .{ }^{13} \mathrm{C} \mathrm{NMR}(125.7 \mathrm{MHz}, \mathrm{CDCl} 3): \delta=24.20,24.92$, 25.47, 30.01 and $33.09\left(5 \mathrm{CH}_{2}\right.$ of cyclohexyl), $33.47\left(\mathrm{CH}_{3}\right), 51.64\left(\mathrm{OCH}_{3}\right), 53.02$ $\left(\mathrm{OCH}_{3}\right), 54.58(\mathrm{CH}$ of cyclohexyl), $58.31(\mathrm{~N}=\mathrm{C}=\mathrm{C}), 60.37(\mathrm{CH}), 127.75,128.80,130.08$ and 134.58 (C aromatic), $145.91(\mathrm{~N}=\mathrm{CH}), 159.27(\mathrm{~N}=\mathrm{C}=\mathrm{C}), 161.23(\mathrm{CON}), 167.80$ $(\mathrm{C}=\mathrm{O}), 170.91(\mathrm{C}=\mathrm{O}) \mathrm{ppm}$.

Dimethyl2-[N'-4-chloro-benzylidene- $N$-(4-pyridineoyl)hydrazino]-3-(cyclohexylim inomethylene)succinate (4b)

Yield: 90\%; Yellow oil; IR (KBr) $\left(v_{\max }, \mathrm{cm}^{-1}\right)$ : $2105(\mathrm{~N}=\mathrm{C}=\mathrm{C}), 1734,1667(\mathrm{C}=\mathrm{O})$. Analyses: Calcd. for $\mathrm{C}_{26} \mathrm{H}_{27} \mathrm{ClN}_{4} \mathrm{O}_{5}$ : C, 61.11; H, 5.33; N, 10.96\%. Found: 61.25; H, 5.50; N, 10.81\%. MS (m/z, \%): $510(\mathrm{M}+, 3) .{ }^{1} \mathrm{H}$ NMR $\left(500.1 \mathrm{MHz}, \mathrm{CDCl}_{3}\right): \delta=1.18-$ $1.98\left(10 \mathrm{H}, \mathrm{m}, 5 \mathrm{CH}_{2}\right.$ of cyclohexyl), $3.70\left(3 \mathrm{H}, \mathrm{s}, \mathrm{OCH}_{3}\right), 3.78\left(3 \mathrm{H}, \mathrm{s}, \mathrm{OCH}_{3}\right), 3.84$ (1 $\mathrm{H}, \mathrm{m}, \mathrm{CH}$ of cyclohexyl), $5.61(1 \mathrm{H}, \mathrm{s}, \mathrm{CH}), 7.28-8.51$ (8 H, m ,aromatic), 8.37 (1 H, s, $\mathrm{N}=\mathrm{CH})$ ppm. 13C NMR (125.7MHz, $\left.\mathrm{CDCl}_{3}\right): \delta=24.21,25.08,25.67,30.18$ and 33.36 $\left(5 \mathrm{CH}_{2}\right.$ of cyclohexyl), $51.65\left(\mathrm{OCH}_{3}\right), 52.90\left(\mathrm{OCH}_{3}\right), 54.47(\mathrm{CH}$ of cyclohexyl), 59.88 $(\mathrm{N}=\mathrm{C}=\mathrm{C}), 62.45(\mathrm{CH}), 122.37,128.83,129.24,133.51,136.07,141.75$ and 151.40 (C aromatic), $146.11(\mathrm{~N}=\mathrm{CH}), 164.01(\mathrm{~N}=\mathrm{C}=\mathrm{C}), 167.12(\mathrm{CON}), 168.76(\mathrm{C}=\mathrm{O}), 170.4$ $(\mathrm{C}=\mathrm{O}) \mathrm{ppm}$.

Dimethyl 2-[N'-4-chloro-benzylidene-N-(3-methyl)hydrazino]-3-(cyclohexylimino methylene)succinate $(\mathbf{4 c})$

Yield: 87\%; Yellow oil; IR ( $\mathrm{KBr})\left(v_{\max }, \mathrm{cm}^{-1}\right)$ : $2065(\mathrm{~N}=\mathrm{C}=\mathrm{C}), 1740,1686(\mathrm{C}=\mathrm{O})$. Analyses: Calcd. for $\mathrm{C}_{22} \mathrm{H}_{26} \mathrm{ClN}_{3} \mathrm{O}_{5}$ : C, 58.99; H, 5.85; N, 9.38\%. Found: 58.80; H, 5.69; N, 9.48\%. MS (m/z, \%): $447(\mathrm{M}+, 9) .{ }^{1} \mathrm{H}$ NMR $\left(500.1 \mathrm{MHz}, \mathrm{CDCl}_{3}\right): \delta=1.21-2.13\left(10 \mathrm{H}, \mathrm{m}, 5 \mathrm{CH}_{2}\right.$ of cyclohexyl), $2.37\left(3 \mathrm{H}, \mathrm{s}, \mathrm{CH}_{3}\right), 3.69\left(3 \mathrm{H}, \mathrm{s}, \mathrm{OCH}_{3}\right), 3.73\left(3 \mathrm{H}, \mathrm{s}, \mathrm{OCH}_{3}\right), 3.80(1 \mathrm{H}, \mathrm{m}, \mathrm{CH}$ of cyclohexyl), $5.62(1 \mathrm{H}, \mathrm{s}, \mathrm{CH}), 7.33(2 \mathrm{H}, \mathrm{d}, 3 \mathrm{JHH}=7 \mathrm{HZ}$, aromatic), $7.61(2 \mathrm{H}, \mathrm{d}, 3 \mathrm{JHH}$ $=7 \mathrm{HZ}$, aromatic), $8.00(1 \mathrm{H}, \mathrm{s}, \mathrm{N}=\mathrm{CH}) \mathrm{ppm} .{ }^{13} \mathrm{C} \mathrm{NMR}\left(125.7 \mathrm{MHz}, \mathrm{CDCl}_{3}\right): \delta=24.24$, 24.99, 25.52, 30.07 and 33.42 (5 $\mathrm{CH}_{2}$ of cyclohexyl), 33.03 $\left(\mathrm{CH}_{3}\right), 48.79(\mathrm{CH}$ of cyclohexyl), $52.08\left(\mathrm{OCH}_{3}\right), 52.65\left(\mathrm{OCH}_{3}\right), 57.25(\mathrm{~N}=\mathrm{C}=\mathrm{C}), 60.28(\mathrm{CH}), 128.89,129.32,133.55$ and 136.01 (C aromatic), $140.09(\mathrm{~N}=\mathrm{CH}), 164.56(\mathrm{~N}=\mathrm{C}=\mathrm{C}), 168.41(\mathrm{CON}), 169.68(\mathrm{C}=\mathrm{O})$, $171.23(\mathrm{C}=\mathrm{O}) \mathrm{ppm}$. 
Diethyl 2-[N'-4-chloro-benzylidene-N-(4-pyridineoyl)hydrazino]-3-(cyclohexylim inomethylene)succinate (4d)

Yield: 93\%; Yellow oil; IR (KBr) $\left(v_{\max }, \mathrm{cm}^{-1}\right)$ : $2070(\mathrm{~N}=\mathrm{C}=\mathrm{C}), 1743,1669(\mathrm{C}=\mathrm{O})$. Analyses: Calcd. for $\mathrm{C}_{28} \mathrm{H}_{31} \mathrm{ClN}_{4} \mathrm{O}_{5}$ : C, 62.39; H, 5.80; N, 10.39\%. Found: C, 62.20; H, 5.93; N, 10.58\%. MS (m/z, \%): $538(\mathrm{M}+, 3) .{ }^{1} \mathrm{H}$ NMR $\left(500.1 \mathrm{MHz}, \mathrm{CDCl}_{3}\right): \delta=1.22-2.01\left(10 \mathrm{H}, \mathrm{m}, 5 \mathrm{CH}_{2}\right.$ of cyclohexyl), $\delta=1.14-1.30\left(6 \mathrm{H}, \mathrm{m}, 2 \mathrm{CH}_{3}\right), 3.88(1 \mathrm{H}, \mathrm{m}, \mathrm{CH}$ of cyclohexyl), 4.11- $4.36(4 \mathrm{H}, \mathrm{m}$, $\left.2 \mathrm{OCH}_{2}\right), 5.83(1 \mathrm{H}, \mathrm{s}, \mathrm{CH}), 7.33-8.56\left(8 \mathrm{H}, \mathrm{m}\right.$, aromatic), $8.39(1 \mathrm{H}, \mathrm{s}, \mathrm{N}=\mathrm{CH}) \mathrm{ppm} .{ }^{13} \mathrm{C}$ NMR $\left(125.7 \mathrm{MHz}, \mathrm{CDCl}_{3}\right): \delta=\delta=14.55$ and $14.82\left(2 \mathrm{CH}_{3}\right), 24.28,25.03,25.63,30.27$ and $33.18\left(5 \mathrm{CH}_{2}\right.$ of cyclohexyl), 54.59 (CH of cyclohexyl), $58.32(\mathrm{~N}=\mathrm{C}=\mathrm{C}), 60.44\left(\mathrm{OCH}_{2}\right), 62.58\left(\mathrm{OCH}_{2}\right), 63.08$ (CH), 122.41, 128.81, 129.20, 133.45, 136.05, 141.77 and 151.35 (C aromatic), $145.69(\mathrm{~N}=\mathrm{CH})$, $164.07(\mathrm{~N}=\mathrm{C}=\mathrm{C}), 167.10(\mathrm{CON}), 168.74(\mathrm{C}=\mathrm{O}), 170.6(\mathrm{C}=\mathrm{O}) \mathrm{ppm}$.

\section{Di-t-butyl2-[N'-4-chloro-benzylidene-N-(3-methyl)hydrazino]-3-(cyclohexylimino methylene)succinate (4e)}

Yield: 94\%; White powder; m.p. 162-164 ${ }^{\circ} \mathrm{C}$. IR $(\mathrm{KBr})\left(v_{\max }, \mathrm{cm}^{-1}\right): 2060(\mathrm{~N}=\mathrm{C}=\mathrm{C}), 1730$, 1688 (C=O). Analyses: Calcd. for $\mathrm{C}_{27} \mathrm{H}_{38} \mathrm{ClN}_{3} \mathrm{O}_{5}$ : C, 62.36; H, 7.36; N, 8.08\%. Found: C, 62.50; H, 7.22; N, 8.24\%. MS (m/z, \%): $519(\mathrm{M}+, 10) .{ }^{1} \mathrm{H}$ NMR (500.1 MHz, $\left.\mathrm{CDCl}_{3}\right)$ : $\delta=1.42-1.99\left(10 \mathrm{H}, \mathrm{m}, 5 \mathrm{CH}_{2}\right.$ of cyclohexyl), $1.42\left(\mathrm{~s}, 9 \mathrm{H}, 3 \mathrm{CH}_{3}\right.$ of t-Bu), 1.45 (s, $9 \mathrm{H}, 3 \mathrm{CH}_{3}$ of t-Bu), 2.39 (3H, s, $\left.\mathrm{CH}_{3}\right), 3.80(1 \mathrm{H}, \mathrm{m}, \mathrm{CH}$ of cyclohexyl), $5.60(1 \mathrm{H}, \mathrm{s}, \mathrm{CH}), 7.36(2 \mathrm{H}, \mathrm{d}$, 3J $=7 \mathrm{~Hz}$, aromatic), $7.62\left(2 \mathrm{H}, \mathrm{d}, 3 \mathrm{~J}=7 \mathrm{~Hz}\right.$, aromatic), $7.96(1 \mathrm{H}, \mathrm{s}, \mathrm{N}=\mathrm{CH}) \mathrm{ppm} .{ }^{13} \mathrm{C} \mathrm{NMR}$ (125.7MHz, $\left.\mathrm{CDCl}_{3}\right): \delta=22.45,24.72,25.74,30.55$ and $33.71\left(5 \mathrm{CH}_{2}\right.$ of cyclohexyl), 28.29 and $28.86\left(6 \mathrm{CH}_{3}\right.$ of $\left.2 \mathrm{t}-\mathrm{Bu}\right), 33.51\left(\mathrm{CH}_{3}\right), 54.18(\mathrm{CH}$ of cyclohexyl), $58.27(\mathrm{~N}=\mathrm{C}=\mathrm{C}), 60.81$ $(\mathrm{CH}), 80.72$ and $82.59\left(2 \mathrm{O}-\mathrm{C}\left(\mathrm{CH}_{3}\right)_{3}\right), 128.76,129.33,133.91$ and 135.80 (C aromatic), 146.17 $(\mathrm{N}=\mathrm{CH}), 159.10(\mathrm{~N}=\mathrm{C}=\mathrm{C}), 165.01(\mathrm{CON}), 166.07(\mathrm{C}=\mathrm{O}), 169.75(\mathrm{C}=\mathrm{O}) \mathrm{ppm}$.

Diethyl2-[N'-4-chloro-benzylidene- $N$-(3-methyl)hydrazino]-3-(tert-butylimino methylene)succinate (4f)

Yield: 88\%; Yellow oil; IR $(\mathrm{KBr})\left(v_{\max }, \mathrm{cm}^{-1}\right): 2065(\mathrm{~N}=\mathrm{C}=\mathrm{C}), 1739,1680(\mathrm{C}=\mathrm{O})$. Anal. Calcd for $\mathrm{C}_{22} \mathrm{H}_{28} \mathrm{ClN}_{3} \mathrm{O}_{5}$ : C, 58.73; H, 6.27; N, 9.34. \%. Found: C, 58.91; H, 6.34; N, 9.23 \%. MS (m/z, \%): $449(\mathrm{M}+, 6) .{ }^{1} \mathrm{H}$ NMR $\left(500 \mathrm{MHz}, \mathrm{CDCl}_{3}\right): \delta=1.23-1.31\left(6 \mathrm{H}, \mathrm{m}, 2 \mathrm{CH}_{3}\right), 1.43\left(9 \mathrm{H}, \mathrm{s}, 3 \mathrm{CH}_{3}\right.$ of t$\mathrm{Bu}), 2.39\left(3 \mathrm{H}, \mathrm{s}, \mathrm{CH}_{3}\right), 4.15-4.30\left(4 \mathrm{H}, \mathrm{m}, 2 \mathrm{OCH}_{2}\right), 5.59(1 \mathrm{H}, \mathrm{s}, \mathrm{CH}), 7.34(2 \mathrm{H}, \mathrm{d}, 3 \mathrm{~J}=7 \mathrm{~Hz}$, aromatic), $7.62\left(2 \mathrm{H}, \mathrm{d}, 3 \mathrm{~J}=7 \mathrm{~Hz}\right.$, aromatic), $7.98(1 \mathrm{H}, \mathrm{s}, \mathrm{N}=\mathrm{CH}) \mathrm{ppm} .{ }^{13} \mathrm{C}$ NMR $(125.7 \mathrm{MHz}$, $\left.\mathrm{CDCl}_{3}\right): \delta=14.53$ and $14.75\left(2 \mathrm{CH}_{3}\right), 22.09\left(\mathrm{CH}_{3}\right), 30.77\left(3 \mathrm{CH}_{3}\right.$ of t-Bu), $53.35(\mathrm{C}), 60.81$ and $62.38\left(2 \mathrm{OCH}_{2}\right), 62.78(\mathrm{~N}=\mathrm{C}=\mathrm{C}), 63.39(\mathrm{CH}), 128.86,129.32,133.64$ and 135.96 (C aromatic), $139.83(\mathrm{~N}=\mathrm{CH}), 164.43(\mathrm{~N}=\mathrm{C}=\mathrm{C}), 167.80(\mathrm{CON}), 170.62(\mathrm{C}=\mathrm{O}), 172.91(\mathrm{C}=\mathrm{O}) \mathrm{ppm}$.

Diethyl 2-[N'-4-chloro-benzylidene-N-(3-methyl)hydrazino]-3-(cyclohexylimino methylene)succinate $\mathbf{( 4 g )}$

Yield: 90\%; Yellow oil; IR ( $\mathrm{KBr})\left(v_{\max }, \mathrm{cm}^{-1}\right)$ : $2084(\mathrm{~N}=\mathrm{C}=\mathrm{C}), 1745,1668(\mathrm{C}=\mathrm{O})$. Analyses: Calcd. for $\mathrm{C}_{24} \mathrm{H}_{30} \mathrm{ClN}_{3} \mathrm{O}_{5}$ : C, 60.56; H, 6.35; N, 8.83\%. Found: 60.75; H, 6.20; N, 8.99\%. MS (m/z, \%): $475(\mathrm{M}+, 3) .{ }^{1} \mathrm{H}$ NMR $\left(500.1 \mathrm{MHz}, \mathrm{CDCl}_{3}\right): \delta=1.17-1.33\left(6 \mathrm{H}, \mathrm{m}, 2 \mathrm{CH}_{3}\right)$, 1.25-1.99 $\left(10 \mathrm{H}, \mathrm{m}, 5 \mathrm{CH}_{2}\right.$ of cyclohexyl), $2.38\left(3 \mathrm{H}, \mathrm{s}, \mathrm{CH}_{3}\right), 3.86(1 \mathrm{H}, \mathrm{m}, \mathrm{CH}$ of cyclohexyl), $4.10-4.28\left(4 \mathrm{H}, \mathrm{m}, 2 \mathrm{OCH}_{2}\right), 5.62(1 \mathrm{H}, \mathrm{s}, \mathrm{CH}), 7.34(2 \mathrm{H}, \mathrm{d}, 3 \mathrm{JHH}=7 \mathrm{HZ}$, aromatic), $7.62(2 \mathrm{H}, \mathrm{d}, 3 \mathrm{JHH}=7 \mathrm{HZ}$, aromatic $), 8.03(1 \mathrm{H}, \mathrm{s}, \mathrm{N}=\mathrm{CH}) \mathrm{ppm} .{ }^{13} \mathrm{C} \mathrm{NMR}$ $\left(125.7 \mathrm{MHz}, \mathrm{CDCl}_{3}\right): \delta=14.55$ and $14.82\left(2 \mathrm{CH}_{3}\right), 22.16\left(\mathrm{CH}_{3}\right), 24.13,25.59,25.68,30.10$ and $33.39\left(5 \mathrm{CH}_{2}\right.$ of cyclohexyl), $53.52(\mathrm{CH}$ of cyclohexyl), $59.28(\mathrm{~N}=\mathrm{C}=\mathrm{C}), 60.80$ and $62.42\left(2 \mathrm{OCH}_{2}\right), 61.66(\mathrm{CH}), 128.87,129.32,133.66$ and 135.95 (C aromatic), 140.01 $(\mathrm{N}=\mathrm{CH}), 167.88(\mathrm{~N}=\mathrm{C}=\mathrm{C}), 169.34(\mathrm{CON}), 170.68(\mathrm{C}=\mathrm{O}), 173.03(\mathrm{C}=\mathrm{O}) \mathrm{ppm}$. 
Diethyl 2-[N'-benzylidene-N-(3-methyl)hydrazino]-3-(cyclohexyliminomethylene) succinate (4h)

Yield: 92\%; Yellow oil; IR $(\mathrm{KBr})\left(v_{\max }, \mathrm{cm}^{-1}\right): 2060(\mathrm{~N}=\mathrm{C}=\mathrm{C}), 1737,1687(\mathrm{C}=\mathrm{O})$. Analyses: Calcd. for $\mathrm{C}_{24} \mathrm{H}_{31} \mathrm{~N}_{3} \mathrm{O}_{5}$ : C, 65.29; H, 7.08; N, 9.52\%. Found: 62.40; H, 7.23; N, 9.41\%. MS (m/z, \%): $441(\mathrm{M}+, 7) .{ }^{1} \mathrm{H}$ NMR (500.1 MHz, $\left.\mathrm{CDCl}_{3}\right): \delta=1.14-1.29(6 \mathrm{H}, \mathrm{m}$, $\left.2 \mathrm{CH}_{3}\right), 1.47-1.92\left(10 \mathrm{H}, \mathrm{m}, 5 \mathrm{CH}_{2}\right.$ of cyclohexyl), $2.04\left(3 \mathrm{H}, \mathrm{s}, \mathrm{CH}_{3}\right), 3.87(1 \mathrm{H}, \mathrm{m}, \mathrm{CH}$ of cyclohexyl), $4.04-4.24\left(4 \mathrm{H}, \mathrm{m}, 2 \mathrm{OCH}_{2}\right), 5.46(1 \mathrm{H}, \mathrm{s}, \mathrm{CH}), 7.08-7.64$ (5 H, m ,aromatic), $7.96(1 \mathrm{H}, \mathrm{s}, \mathrm{N}=\mathrm{CH}) \mathrm{ppm} .{ }^{13} \mathrm{C} \mathrm{NMR}\left(125.7 \mathrm{MHz}, \mathrm{CDCl}_{3}\right): \delta=14.57$ and $14.71\left(2 \mathrm{CH}_{3}\right), 22.05$ $\left(\mathrm{CH}_{3}\right), 24.19,25.53,25.81,29.97$ and $33.37\left(5 \mathrm{CH}_{2}\right.$ of cyclohexyl), $54.20(\mathrm{CH}$ of cyclohexyl), $59.93(\mathrm{~N}=\mathrm{C}=\mathrm{C}), 61.70$ and $62.80\left(2 \mathrm{OCH}_{2}\right), 62.38(\mathrm{CH}), 128.92,129.47,131.23$ and 134.45(C aromatic), $146.81(\mathrm{~N}=\mathrm{CH}), 159.31(\mathrm{~N}=\mathrm{C}=\mathrm{C}), 161.90(\mathrm{CON}), 168.03(\mathrm{C}=\mathrm{O})$, $170.91(\mathrm{C}=\mathrm{O}) \mathrm{ppm}$.

\section{Results and Discussion}

Thus, alkyl isocyanides $\mathbf{1}$ and dialkyl acetylenedicarboxylates (2) in the presence of carboxylic acid arylidene-hydrazides (3) undergo a smooth 1:1:1 addition reaction in acetone at ambient temperature to produce highly functionalized ketenimines (4a-h) in excellent yields (Figure 1).

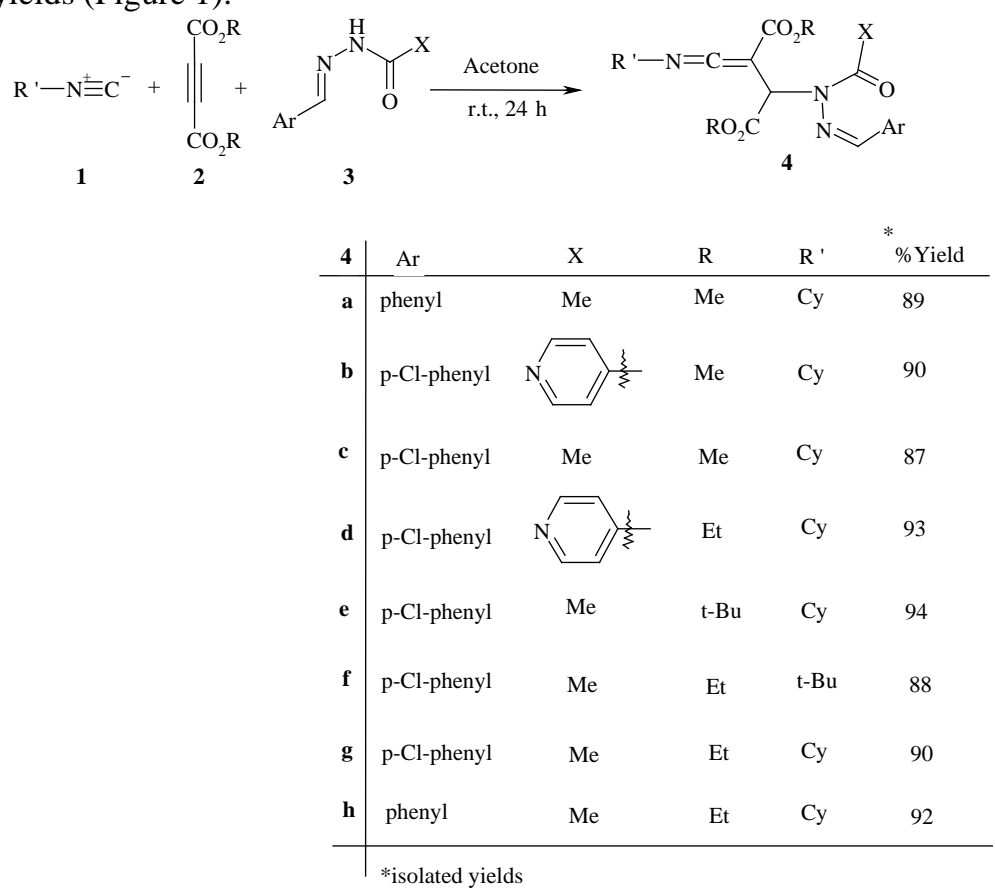

Figure 1. Three-component reaction between alkyl isocyanides and dialkyl acetylenedicarboxylates in the presence of carboxylic acid arylidene-hydrazides

The structures of compounds $\mathbf{4 a - h}$ were deduced from their elemental analyses and their IR, ${ }^{1} \mathrm{H}$ NMR, ${ }^{13} \mathrm{C}$ NMR spectra. The IR spectrum of $\mathbf{4 e}$ exhibited the absorption band for the ketenimine moiety at $2060 \mathrm{~cm}^{-1}$ and for the ester carbonyl groups at 1730 and $1688 \mathrm{~cm}^{-1}$.

The ${ }^{1} \mathrm{H}$ NMR spectrum of compound $4 \mathrm{e}$ exhibited three sharp singlet signals readily recognized as arising from methyl groups of the t-Bu $(\delta=1.42$ and 1.45) and the protons of 
the methyl was appeared at $2.39 \mathrm{ppm}$. The proton $\mathrm{CH}$ was appeared at $\delta=5.60 \mathrm{ppm}$. The $\mathrm{NCH}$ proton was appeared as a multiplet at $3.80 \mathrm{ppm}$ and the signals related to methylene groups of cyclohexyl moiety were observed as multiplets at $1.42-1.99 \mathrm{ppm}$. A single signal was observed at $7.96 \mathrm{ppm}$ which arises from $\mathrm{N}=\mathrm{CH}$ proton. The ${ }^{13} \mathrm{C}$ NMR spectrum of compound 4e showed $\mathbf{2 2}$ distinct resonances in agreement with the proposed structure. The $\mathrm{sp}^{2}$ - hybridized carbon atom of the ketenimine residue appears at $\delta=58.27 \mathrm{ppm}$, as a result of strong electron delocalization. Partial assignments of these resonances are given in the experimental section.

A plausible mechanism for the formation of ketenimine 4a-h is shown in Figure 2. On the basis of the well-established chemistry of isocyanides, $\mathbf{1 , 2 , 6 , 7}$ it is reasonable to assume that the functionalized ketenimine $\mathbf{4}$ results from the initial addition of the isocyanide to the dialkyl acetylenedicarboxylate and subsequent protonation of the 1:1 adduct 5 by carboxylic acid arylidene-hydrazide. Then, the positively charged ion 6 is attacked by anion 7 to give the product 4 (Figure 2).
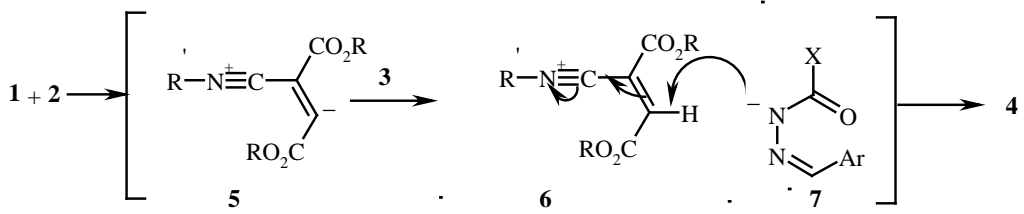

Figure 2. Suggested mechanism for formation compound 4

The present method carries the advantage that, not only is the reaction performed under neutral conditions, but the educts can be mixed without any activation or modification.

\section{Conclusion}

In summary, the simple one-pot reaction between alkyl isocyanides and dialkyl acetylenedicarboxylates in the presence of carboxylic acid arylidene-hydrazides provides access to stable ketenimine derivatives of potential synthetic interest in high yields.

\section{Acknowledgement}

We gratefully acknowledge financial support from the Research Council of Islamic Azad University of Zahedan and The Islamic Azad University of Yazd of Iran.

\section{References}

1. Alajarín M, Vidal A and Tovar F, Targets Heterocycl Syst., 2000, 4, 293.

2. Patai S, The Chemistry of Ketenes Allenes and Related Compounds. Wiley, New York, 1980.

3. Schmittel M, Steffen J P, Angel M A W, Engels B, Lennartz C and Hanrath M, Angew Chem Int Ed., 1998, 37, 1562.

4. Alajarin M, Vidal A, Tovar F and Arellano M C R, Tetrahedron: Asymmetry, 2004, 15, 489.

5. $\quad$ Calter M A, J Org Chem., 1996, 61, 8006.

6. Ugi I, Angew Chem Int Ed., 1982, 21, 810.

7. $\quad$ Domling A and Ugi I, Angew Chem Int Ed., 2000, 39, 3168.

8. Dömling A, Chem Rev., 2006, 106, 17.

9. Marcaccini S and Torroba T, Org Prep Proced Int., 1993, 25, 141.

10. Shaabani A, Soleimani E, Khavasi H R, Hoffmann R D, Rodewald U C and Po" ttgen R, Tetrahedron Lett., 2006, 47, 5493. 
11. Yavari I, Zare H and Mohtat B, Mol Diversity, 2006, 10, 24.

12. Yavari I, Nasiri F and Djahaniani H, Mol Diversity, 2004, 8, 431.

13. Yavari I, Alizadeh A, Anary-Abbasinejad M and Bijanzadeh H R, Tetrahedron, 2003, 59, 6083.

14. Shaabani A, Teimouri M B and Arab-Ameri S, Tetrahedron Lett., 2004, 45, 8409.

15. Yavari I, Anary-Abbasinejad M, Alizadeh A and Hossaini Z, Tetrahedron, 2003, 59, 1289.

16. Anary-Abbasinejad M, Mosslemin M H, Anaraki-Ardakani H and Tahan S, J Chem Res., 2006, 306.

17. Anary-Abbasinejad M, Anaraki-Ardakani H, Rastegari F and Hassanabadi A, J Chem Res., 2007, 602.

18. Anary-Abbasinejad M, Hassanabadi A and Aiinparast F, J Chem Res., 2010, 613.

19. Mosslemin M H, Nateghi M R, Hassanabadi A and Zare M, J Chem Res., 2010, 178. 